Acta Univ. Sapientiae, Informatica 11, 1 (2019) 95-108

DOI: $10.2478 /$ ausi-2019-0007

\title{
J-coloring of graph operations
}

\author{
Sudev NADUVATH \\ Department of Mathematics \\ CHRIST (Deemed to be University \\ Bangalore, INDIA \\ email: sudev.nk@christuniversity.in
}

\author{
Johan KOK \\ Department of Mathematics \\ CHRIST (Deemed to be University \\ Bangalore, INDIA \\ email: jacotype@gmail.com
}

\begin{abstract}
A vertex $v$ of a given graph is said to be in a rainbow neighbourhood of $\mathrm{G}$ if every color class of $\mathrm{G}$ consists of at least one vertex from the closed neighbourhood $\mathrm{N}[v]$. A maximal proper coloring of a graph $\mathrm{G}$ is a J-coloring if and only if every vertex of $\mathrm{G}$ belongs to a rainbow neighbourhood of G. In general all graphs need not have a J-coloring, even though they admit a chromatic coloring. In this paper, we characterise graphs which admit a J-coloring. We also discuss some preliminary results in respect of certain graph operations which admit a J-coloring under certain conditions.
\end{abstract}

\section{Introduction}

For general notations and concepts in graphs and digraphs we refer to $[1,3$, 9]. For further definitions in the theory of graph coloring, see [2, 4]. Unless specified otherwise, all graphs mentioned in this paper are simple, connected and undirected graphs.

The degree of a vertex $v \in V(G)$ is the number of edges in $G$ incident with $v$ and is denoted $\mathrm{d}_{\mathrm{G}}(v)$ or when the context is clear, simply as $\mathrm{d}(v)$. A pendant vertex or an end vertex of a graph $\mathrm{G}$ is a vertex having degree 1 . A vertex

Computing Classification System 1998: G.2.2

Mathematics Subject Classification 2010: 05C15, 05C38, 05C75, 05C85.

Key words and phrases: rainbow neighbourhood, J-coloring, J*-coloring. 
which is not a pendant vertex is called an internal vertex of $\mathrm{G}$ (see [3]). A pendant edge of $\mathrm{G}$ is an edge incident on a pendant vertex of $\mathrm{G}$. Also, unless mentioned otherwise, the graphs we consider in this paper has the order $n$ and size $p$ with minimum and maximum degree $\delta$ and $\Delta$, respectively.

Recall that if $\mathcal{C}=\left\{c_{1}, c_{2}, c_{3}, \ldots, c_{\ell}\right\}$ and $\ell$ sufficiently large, is a set of distinct colors, a proper vertex coloring of a graph $\mathrm{G}$ is a vertex coloring $\varphi: \mathrm{V}(\mathrm{G}) \mapsto \mathcal{C}$ such that no two distinct adjacent vertices have the same color. The cardinality of a minimum set of colors which allows a proper vertex coloring of $G$ is called the chromatic number of $\mathrm{G}$ and is denoted by $\chi(\mathrm{G})$. When a vertex coloring is considered with colors of minimum subscripts, the coloring is called a minimum parameter coloring. Unless stated otherwise, all colorings in this paper are minimum parameter color sets.

The number of times a color $c_{i}$ is allocated to vertices of a graph $G$ is denoted by $\theta\left(c_{i}\right)$ and $\varphi: v_{i} \mapsto c_{j}$ is abbreviated, $c\left(v_{i}\right)=c_{j}$. Furthermore, if $\mathrm{c}\left(v_{i}\right)=\mathrm{c}_{j}$ then $\mathrm{l}\left(v_{i}\right)=\mathrm{j}$. The color class of a color $\mathrm{c}_{i}$, denoted by $\mathcal{C}_{\mathfrak{i}}$, is the set of vertices of $G$ having the same color $c_{i}$.

We shall also color a graph in accordance with the rainbow neighbourhood convention (see [5]), which is stated as follows.

Rainbow neighbourhood convention: ([5]) For a proper coloring $\mathcal{C}=$ $\left\{c_{1}, c_{2}, c_{3}, \ldots, c_{\ell}\right\}, \chi(G)=\ell$, we always color maximum possible number of vertices with the color $c_{1}$, then color the maximum possible number of remaining vertices by the color $c_{2}$ and proceeding like this and finally color the remaining vertices by the color $\mathfrak{c}_{\ell}$. Such a coloring is called a $\chi^{-}$-coloring of a graph.

The inverse to the convention requires the mapping $c_{j} \mapsto c_{\ell-(j-1)}$. Corresponding to the inverse coloring we define $\iota^{\prime}\left(v_{i}\right)=\ell-(j-1)$ if $c\left(v_{i}\right)=c_{j}$. The inverse of a $\chi^{-}$-coloring is called a $\chi^{+}$-coloring.

The closed neighbourhood $N[v]$ of a vertex $v \in V(G)$ which contains at least one colored vertex of each color in the chromatic coloring, is called a rainbow neighbourhood. That is, a vertex $\mathrm{V}$ is said to be in a rainbow neighbourhood if $\mathcal{C}_{i} \cap \mathrm{N}[v] \neq \emptyset$, for all $1 \leq i \leq \chi(\mathrm{G})$. The number of vertices of a graph $\mathrm{G}$, which belong to some rainbow neighbourhoods of $\mathrm{G}$ is called the rainbow neighbourhood number of $\mathrm{G}$, denoted by $\mathrm{r}_{\chi(\mathrm{G})}$ (see [5]). The rainbow neighbourhood number of certain graph classes have been determined in $[6,7]$.

Motivated by these studies, two types of vertex colorings in terms of rainbow neighbourhoods have been introduced in [8] as follows.

Definition 1 [8] A maximal proper coloring of a graph $\mathrm{G}$ is a Johan coloring of $\mathrm{G}$, or J-coloring in short, if and only if every vertex of $\mathrm{G}$ belongs to a rainbow neighbourhood of $\mathrm{G}$. The maximum number of colors in a J-coloring 
is called the J-chromatic number of $\mathrm{G}$, denoted by $\mathcal{J}(\mathrm{G})$.

Definition 2 [8] A maximal proper coloring of a graph $\mathrm{G}$ is a modified Johan coloring, or $\mathrm{J}^{*}$-coloring in short, if and only if every internal vertex (a vertex having degree at least 2) of $G$ belongs to a rainbow neighbourhood of $G$. The maximum number of colors in a $J^{*}$-coloring is denoted by $J^{*}(G)$.

Figure 1 illustrate a J-colorable and a J*-colorable graph.

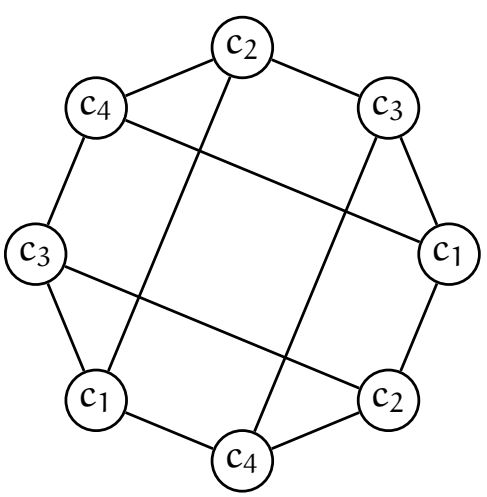

(a) A graph with a J-coloring.



(b) A graph with a J*-coloring.

Figure 1: J-colorable and $\mathrm{J}^{*}$ colorable graphs

In this paper, we characterise the graphs which admit J-coloring. We also discuss some preliminary results in respect of certain graph operations which admit a J-coloring under certain conditions.

\section{Results and discussions}

A null graph on $\mathrm{n}$ vertices is an edgeless graph and is denoted by $\mathfrak{N}_{n}$. We follow the convention that $\mathcal{J}\left(\mathfrak{N}_{n}\right)=\mathcal{J}^{*}\left(\mathfrak{N}_{\mathfrak{n}}\right)=1, n \in \mathbb{N}$. Also, note that for any graph $G$ which admits a J-coloring, we have $\chi(G) \leq \mathcal{J}(G)$.

Note that if a graph $G$ admits a J-coloring, it also admits a $J^{*}$-coloring. However, the converse need not be true always. It can also be noted that if graph $G$ has no pendant vertex and it admits a J-coloring, then $\mathcal{J}(G)=\mathcal{J}^{*}(G)$.

In view of the above mentioned concepts and facts, we have the following theorem. 
Theorem 3 If $\mathrm{G}$ is a tree of order $\mathrm{n} \geq 2$, then $\mathcal{J}(\mathrm{G})<\mathcal{J}^{*}(\mathrm{G})$.

Proof. A tree $\mathrm{G}$ of order $\mathrm{n} \geq 2$ has at least two pendant vertices, say $\boldsymbol{u}$ and $v$. Therefore, the maximum number of colors which will allow both vertices $u$ and $v$ to yield rainbow neighbourhoods is $\chi(G)=2$. Therefore, $G$ admits a J-coloring and $\mathcal{J}(\mathrm{G})=2$.

Any internal vertex $w$ of $G$ has $d(w) \geq 2$. Therefore, $\mathcal{J}^{*}(G) \leq 3$. Consider any diameter path of $G$ say $P_{\operatorname{diam}(G)}$. Beginning at a pendant vertex of the diameter path, label the vertices consecutively $v_{1}, v_{2}, v_{3}, \ldots, v_{\text {diam }(G)}$. color the vertices consecutively $\mathrm{c}\left(v_{1}\right)=\mathrm{c}_{1}, \mathrm{c}\left(v_{2}\right)=\mathrm{c}_{2}, \mathrm{c}\left(v_{3}\right)=\mathrm{c}_{3}, \mathrm{c}\left(v_{4}\right)=\mathrm{c}_{1}, \mathrm{c}\left(v_{5}\right)=\mathrm{c}_{2}$, $\mathrm{c}\left(v_{6}\right)=\mathrm{c}_{3}$ and so on such that

$$
\begin{array}{ll}
c\left(v_{\operatorname{diam}(G)}\right)=1 ; & \text { if } \operatorname{diam}(G) \equiv 1(\bmod 3) \\
c\left(v_{\operatorname{diam}(G)}\right)=2 ; & \text { if } \operatorname{diam}(G) \equiv 2(\bmod 3) \\
c\left(v_{\operatorname{diam}(G)}\right)=3 ; & \text { if } \operatorname{diam}(G) \equiv 0(\bmod 3) .
\end{array}
$$

Clearly, in respect of path $P_{\operatorname{diam}(G)}$, it is a proper coloring and all internal vertices yield a rainbow neighbourhood on 3 colors. Consider any maximal path starting from, say $v \in \mathrm{V}\left(\mathrm{P}_{\operatorname{diam}(\mathrm{G})}\right)$. Hence, $v$ is a pendant vertex to that maximal path. color the vertices consecutively from $v$ as follows:

(a) If $c(v)=c_{1}$ in $P_{\operatorname{diam}(G)}$, color as $c_{1}, c_{2}, c_{3}, c_{1}, c_{2}, c_{3}, \ldots, \underbrace{c_{1} \text { or } c_{2} \text { or } c_{3}}$

(b) If $c(v)=c_{2}$ in $P_{\operatorname{diam}(G)}$, color as $c_{2}, c_{3}, c_{1}, c_{2}, c_{3}, c_{1}, \cdots, \underbrace{c_{2} \text { or } c_{3} \text { or } c_{1}}$.

(c) If $c(v)=c_{3}$ in $P_{\operatorname{diam}(G)}$, color as $c_{3}, c_{1}, c_{2}, c_{3}, c_{1}, c_{2}, \cdots, \underbrace{c_{3} \text { or } c_{1} \text { or } c_{2}}$.

It follows from mathematical induction that all maximal branching can receive such coloring which remains a proper coloring with all internal vertices $v \in \mathrm{V}(\mathrm{G})$ having $|\mathrm{c}(\mathrm{N}[v])|=3$. Furthermore, all nested branching can be colored in a similar way until all vertices of $G$ are colored. Therefore, $\mathcal{J}^{*}(G) \geq 3$. Hence, $\mathcal{J}(G)<\mathcal{J}^{*}(G)$.

An easy example to illustrate Theorem 3 is the star $K_{1, n}, n \geq 2$ for which $\mathcal{J}\left(K_{1, n}\right)=2<n+1=\mathcal{J}^{*}\left(K_{1, n}\right)$. This example prompts the next results.

Corollary 4 For any graph $\mathrm{G}$ which admits a $\mathrm{J}^{*}$-coloring, we have $\mathrm{J}^{*}(\mathrm{G}) \leq$ $\Delta(\mathrm{G})+1$. 
Corollary 5 If $\mathcal{J}^{*}(\mathrm{G})>\mathcal{J}(\mathrm{G})$ for a graph $\mathrm{G}$, then $\mathrm{G}$ has at least one pendant vertex.

Proof. Since all $v \in V(G)$ are internal vertices and any vertex $u$ for which $\mathrm{d}(\mathrm{u})=\delta(\mathrm{G})$ must yield a rainbow neighbourhood, it follows that any maximal proper coloring $\mathcal{C}$ are bound to $|\mathcal{C}|=|N[u]|=\delta(G)+1$. Therefore, if $\mathcal{J}^{*}(\mathrm{G})>$ $\mathcal{J}(\mathrm{G})$, then $\mathrm{G}$ has at least one pendant vertex.

In [5], the rainbow neighbourhood number $r_{\chi}(G)$ is defined as the number of vertices of $\mathrm{G}$ which yield rainbow neighbourhoods. It is evident that not all graphs admit a J-coloring. Then, we have

Lemma 6 (i) A maximal proper coloring $\varphi: \mathrm{V}(\mathrm{G}) \mapsto \mathcal{C}$ of a graph $\mathrm{G}$ which satisfies a graph theoretical property, say $\mathfrak{P}$, can be minimised to obtain a minimal proper coloring which satisfies $\mathfrak{P}$.

(ii) A minimal proper coloring $\varphi: \mathrm{V}(\mathrm{G}) \mapsto \mathcal{C}$ of a graph $\mathrm{G}$ which satisfies a graph theoretical property, say $\mathfrak{P}$, can be maximised to obtain a maximal proper coloring which satisfies $\mathfrak{P}$.

\section{Proof.}

(i) Consider a maximal proper coloring $\varphi: \mathrm{V}(\mathrm{G}) \mapsto \mathcal{C}$ of a graph $\mathrm{G}$ which satisfies a graph theoretical property say, $\mathfrak{P}$. If a minimum color set $\mathcal{C}^{\prime}$, with $\left|\mathcal{C}^{\prime}\right|<|\mathcal{C}|$, such that a minimal proper coloring $\varphi^{\prime}: \mathrm{V}(\mathrm{G}) \mapsto \mathcal{C}^{\prime}$ which satisfies the graph theoretical property $\mathfrak{P}$ cannot be found, then $|\mathcal{C}|$ is minimum.

(ii) Consider a minimal proper coloring $\varphi: \mathrm{V}(\mathrm{G}) \mapsto \mathcal{C}$ of a graph $\mathrm{G}$ which satisfies a graph theoretical property say, $\mathfrak{P}$. If a maximum color set $\mathcal{C}^{\prime}$, $\left|\mathcal{C}^{\prime}\right|>|\mathcal{C}|$, such that a maximal proper coloring $\varphi^{\prime}: \mathrm{V}(\mathrm{G}) \mapsto \mathcal{C}^{\prime}$ which satisfies the graph theoretical property $\mathfrak{P}$ cannot be found, then $|\mathcal{C}|$ is maximum.

The following theorem characterises those graphs which admit a J-coloring.

Theorem 7 A graph $\mathrm{G}$ of order $\mathrm{n}$ admits a J-coloring if and only if $\mathrm{r}_{\chi}(\mathrm{G})=$ n.

Proof. If $r_{\chi}(G)=n$, then every vertex of $G$ belongs to a rainbow neighbourhood. Hence, either the chromatic coloring $\varphi: \mathrm{V}(\mathrm{G}) \mapsto \mathcal{C}$ is maximal or a maximal coloring $\varphi^{\prime}: \mathrm{V}(\mathrm{G}) \mapsto \mathcal{C}^{\prime}$ exists. 
An immediate consequence of Definition 1 is that if graph $G$ admits a Jcoloring then each vertex $v \in \mathrm{V}(\mathrm{G})$ yields a rainbow neighbourhood. This consequence also follows from the the result that for any connected graph $G$, $\mathcal{J}(\mathrm{G}) \leq \delta(\mathrm{G})+1$ (see [8]). Hence, from Lemma 6 it follows that either the J-coloring is minimal or a minimal coloring $\varphi^{\prime}: \mathrm{V}(\mathrm{G}) \mapsto \mathcal{C}^{\prime}$ exists such that $r_{\chi}(G)=n$

The following theorem establishes a necessary and sufficient condition for a graph $\mathrm{G}$ to have a J-coloring with respect to a $\chi^{-}$-coloring of $\mathrm{G}$.

Theorem 8 A graph $\mathrm{G}$ admits a J-coloring if and only if each $v \in \mathrm{V}(\mathrm{G})$ yields a rainbow neighbourhood with respect to a $\chi^{-}$-coloring of $\mathrm{G}$.

Proof. If in a $\chi^{-}$-coloring of $\mathrm{G}$, each $v \in \mathrm{V}(\mathrm{G})$ yields a rainbow neighbourhood it follows from the second part of Lemma 6 that the corresponding proper coloring can be maximised to obtain a J-coloring.

Conversely, assume that a graph $G$ admits a J-coloring. Then, it follows from Lemma 6(i) that the corresponding proper coloring can be minimised to obtain a minimal proper coloring for which each $v \in \mathrm{V}(\mathrm{G})$ yields a rainbow neighbourhood. Let the aforesaid set of colors be $\mathcal{C}^{\prime}$. Assume that a minimum set of colors $\mathcal{C}$ exists which is a $\chi^{-}$-coloring of $\mathrm{G}$ and $|\mathcal{C}|<\left|\mathcal{C}^{\prime}\right|$. It implies that there exists at least one vertex $v \in V(G)$ for which at least one distinct pair of vertices, say $u, w \in N(v)$ exists such that $u$ and $v$ are non-adjacent. Furthermore, $\mathrm{c}(\mathrm{u})=\mathrm{c}(\boldsymbol{w})$ under the coloring $\varphi: \mathrm{V}(\mathrm{G}) \mapsto \mathcal{C}$.

Assume that there is exactly one such $v$ and exactly one such vertex pair $u, w \in N(v)$. But then both $u$ and $w$ yield rainbow neighbourhoods in $G$ under the proper coloring $\varphi: \mathrm{V}(\mathrm{G}) \mapsto \mathcal{C}$, which is a contradiction to the minimality of $\mathcal{C}^{\prime}$. By mathematical induction, similar contradictions arise for all vertices similar to $v$. This completes the proof.

\section{Analysis for certain graphs}

Note that we have two types of operations related to graphs, that is: operations on a graph $G$ and operations between two graphs $G$ and $H$. Operations on a graph $G$ result in a well defined derivative of $G$. Examples are the complement graph $G^{c}$, the line graph $L(G)$, the middle graph $M(G)$, the central graph $\mathrm{C}(\mathrm{G})$, the jump graph $\mathrm{J}(\mathrm{G})$ and the total graph $\mathrm{T}(\mathrm{G})$ and so on. Recall that the jump graph $J(G)$ of a graph $G$ of order $n \geq 3$ is the complement graph of the line graph $\mathrm{L}(\mathrm{G})$. Also, note that the line graph is the graphical realisation of edge adjacency in $\mathrm{G}$ and the jump graph is the graphical realisation of edge 
independence in G. Some other graph derivative operations are edge deletion, vertex deletion, edge contraction, thorning a graph by pendant vertex addition and so on.

Examples of operations between graphs $\mathrm{G}$ and $\mathrm{H}$ are, the corona between $\mathrm{G}$ and $\mathrm{H}$ denoted, $\mathrm{G} \circ \mathrm{H}$, the join denoted, $\mathrm{G}+\mathrm{H}$, the disjoint union denoted, $\mathrm{G} \cup \mathrm{H}$, the Cartesian product denoted, $\mathrm{G} \square \mathrm{H}$ and so on.

\subsection{Operations between certain graphs}

The following result establishes a necessary and sufficient condition for the corona of two graphs $\mathrm{G}$ and $\mathrm{H}$ to admit a J-coloring.

Theorem 9 If graphs $\mathrm{G}$ and $\mathrm{H}$ admit $\mathrm{J}$-colorings, then $\mathrm{G} \circ \mathrm{H}$ admits a Jcoloring if and only if either $\mathrm{G}=\mathrm{K}_{1}$ or $\mathcal{J}(\mathrm{G})=\mathcal{J}(\mathrm{H})+1$.

Proof. Part 1: If $\mathrm{G}=\mathrm{K}_{1}$ assume $\mathcal{C}=\left\{\mathrm{c}_{1}, \mathrm{c}_{2}, \mathrm{c}_{3}, \ldots, \mathrm{c}_{\mathfrak{f}(\mathrm{H})}\right\}$ provides a J-coloring of $\mathrm{H}$. color $\mathrm{K}_{1}$ the color $\mathrm{c}_{\mathfrak{J}(\mathrm{H})+1}$. Clearly, $\mathcal{C}^{\prime}=\mathcal{C} \cup\left\{\mathbf{c}_{\mathfrak{f}(\mathrm{H})+1}\right\}$ is a J-coloring of $\mathrm{K}_{1} \circ \mathrm{H}$.

Part 2: If $\mathrm{G} \neq \mathrm{K}_{1}$ and $\mathcal{J}(\mathrm{G})=\mathcal{J}(\mathrm{H})+1$ let $\mathcal{C}=\left\{\mathrm{c}_{1}, \mathrm{c}_{2}, \mathrm{c}_{3}, \ldots, \mathrm{c}_{\ell}, \ell=\mathcal{J}(\mathrm{G})\right\}$ and $\mathcal{C}^{\prime}=\left\{c_{1}, c_{2}, c_{3}, \ldots, c_{\ell-1}, \ell=\mathcal{J}(G)\right\}$ provide the J-colorings of $G$ and $H$, respectively. Assume that $v \in \mathrm{V}(\mathrm{G})$ has $\mathrm{c}(v)=\mathrm{c}_{\mathfrak{i}}$ then color all $\mathrm{u} \in \mathrm{V}(\mathrm{H})$ for the copy of $\mathrm{H}$ corona'd to $v$ for which $c(u)_{(\text {in } H)}=c_{i}, 1 \leq i \leq \ell$, to be $c_{\ell+1}$. Clearly every vertex $v \in \mathrm{V}(\mathrm{G}) \cup \mathrm{V}(\mathrm{H})$ yields a rainbow neighbourhood and $|\mathcal{C}|$ is maximal.

Conversely, let $\mathrm{G} \circ \mathrm{H}$ admit a J-coloring. Then, for any vertex $v \in \mathrm{V}(\mathrm{G})$ the subgraph $v \circ \mathrm{H}$ holds the condition $\mathrm{c}(v) \neq \mathrm{c}(u), \forall u \in V(H)$. Therefore, either $\mathrm{G}=\mathrm{K}_{1}$ or $\mathcal{J}(\mathrm{G})=\mathcal{J}(\mathrm{H})+1$.

The next corollary requires no proof as it is a direct consequence of Theorem 9 .

Corollary 10 If $\mathrm{G} \circ \mathrm{H}$ admits a J-coloring then: $\mathcal{J}(\mathrm{G} \circ \mathrm{H})=\mathcal{J}(\mathrm{G})$.

The following theorem discusses the admissibility of J-coloring by the join of two graphs.

Theorem 11 The join $\mathrm{G}+\mathrm{H}$ admits a J-coloring if and only if both graphs $\mathrm{G}$ and $\mathrm{H}$ admit a $\mathrm{J}$-coloring.

Proof. Assume that both $\mathrm{G}$ and $\mathrm{H}$ admit a J-coloring. Without loss of generality, let $\mathcal{J}(\mathrm{G}) \leq \mathcal{J}(\mathrm{H})$. Assume that $\varphi: \mathrm{V}(\mathrm{G}) \mapsto \mathcal{C}, \mathcal{C}=\left\{\mathrm{c}_{1}, \mathrm{c}_{2}, \mathrm{c}_{3}, \ldots, \mathrm{c}_{\ell}\right\}$ 
and $\varphi^{\prime}: \mathrm{V}(\mathrm{H}) \mapsto \mathcal{C}^{\prime}, \mathcal{C}^{\prime}=\left\{c_{1}, c_{2}, c_{3}, \ldots, c_{\ell^{\prime}}\right\}$ is a J-coloring of $G$ and $H$, respectively. For each $v \in V(G), c(v)=c_{\mathfrak{i}}$ recolor $c(v) \mapsto c_{i+\ell^{\prime}}$. Denote the new color set by $\mathcal{C}_{i+\ell^{\prime}}$. Clearly, each vertex $v \in \mathrm{V}(\mathrm{G})$ is adjacent to at least one of each color in $\mathrm{G}+\mathrm{H}$ hence, each such vertex yields a rainbow neighbourhood in $\mathrm{G}+\mathrm{H}$. Similarly, each vertex $u \in \mathrm{V}(\mathrm{H})$ is adjacent to at least one of each color in $\mathrm{G}+\mathrm{H}$ and hence each such vertex yields a rainbow neighbourhood in $\mathrm{G}+\mathrm{H}$. Furthermore, since both $|\mathcal{C}|,\left|\mathcal{C}^{\prime}\right|$ is maximal color sets, the set $\left|\mathcal{C}_{i+\ell^{\prime}} \cup \mathcal{C}^{\prime}\right|$ is maximal. Therefore, $\mathrm{G}+\mathrm{H}$ admits a J-coloring.

The converse follows trivially from the fact that the additional edges between $\mathrm{G}$ and $\mathrm{H}$ as defined for join form an edge cut in $\mathrm{G}+\mathrm{H}$.

The following result discusses the existence of a J-coloring for the Cartesian product of two given graphs.

Theorem 12 If graphs $\mathrm{G}$ and $\mathrm{H}$ of order $\mathrm{n}$ and $\mathrm{m}$ respectively admit a Jcoloring, then

(i) $\mathrm{G} \square \mathrm{H}$ admits a J-coloring.

(ii) $\mathcal{J}(\mathrm{G} \square \mathrm{H})=\max \{\mathcal{J}(\mathrm{G}), \mathcal{J}(\mathrm{H})\}$

\section{Proof.}

(i) Without loss of generality assume $\mathcal{J}(\mathrm{H}) \geq \mathcal{J}(\mathrm{G})$. Also, assume that $V(G)=\left\{v_{i}: 1 \leq i \leq n\right\}$ and $V(H)=\left\{u_{i}: 1 \leq i \leq m\right\}$. From the definition of $\mathrm{G} \square \mathrm{H}$ it follows that $\mathrm{V}(\mathrm{G} \square \mathrm{H})=\left\{\left(v_{i}, u_{j}\right): 1 \leq i \leq n, 1 \leq\right.$ $j \leq \mathfrak{m}\}$. For $i=1$, if $u_{j} \sim u_{k}$ in $H$, where $\sim$ denotes the adjacency, then $\left(v_{1}, u_{j}\right) \sim\left(v_{1}, u_{k}\right)$ and hence we obtain an isomorphic copy of $\mathrm{H}$. Such a copy admits a J-coloring identical to that of $\mathrm{H}$ in respect of the vertex elements $\mathfrak{u}_{1}, \mathfrak{u}_{2}, \mathfrak{u}_{3}, \ldots, \mathfrak{u}_{\mathrm{m}}$. Now obtain the disjoint union with the copies of $\mathrm{H}$ corresponding to $i=2,3,4, \ldots, n$. Apply the definition of $\mathrm{G} \square \mathrm{H}$ for $\mathrm{u}_{1}$ and if $v_{i} \sim v_{j}$ in $\mathrm{G}$, then $\left(v_{i}, \mathrm{u}_{1}\right) \sim\left(v_{j}, \mathrm{u}_{1}\right)$. An interconnecting copy of $G$ is obtained which result in the first iteration connected graph. Similarly, this copy of $\mathrm{G}$ admits a J-coloring identical to that of $\mathrm{G}$ in respect of the vertex elements $v_{1}, v_{2}, v_{3}, \ldots, v_{n}$. Proceeding iteratively to add all copies of $\mathrm{G}$ for $i=2,3,4, \ldots, n$ in terms of the definition of $\mathrm{G} \square \mathrm{H}$, clearly shows that a J-coloring is admitted.

(ii) The second part of the result follows from the similar reasoning used to prove and hence, $\chi(\mathrm{G} \square \mathrm{H})=\max \{\chi(\mathrm{G}), \chi(\mathrm{H})\}$. 


\subsection{Operations on certain graphs}

Recall that for any connected graph $G, \mathcal{J}(G) \leq \delta(G)+1$ (see [8]) and for $n \geq 2, \mathcal{J}\left(P_{n}\right)=2$ and $J^{*}\left(P_{n}\right)=3$. In view of these results, we have the following results in respect of certain operations on paths and cycles.

Proposition 13 For a path $\mathrm{P}_{\mathrm{n}}, \mathrm{n} \geq 2$ with edge set consecutively labeled as $\mathrm{e}_{1}, \mathrm{e}_{2}, \mathrm{e}_{3}, \ldots, \mathrm{e}_{\mathrm{n}-1}$ and the corresponding line graph vertices consecutively labeled as $\mathfrak{u}_{1}, \mathfrak{u}_{2}, \mathfrak{u}_{3}, \ldots, \mathfrak{u}_{n-1}$. We have

(i) $\mathcal{J}\left(\mathrm{L}\left(\mathrm{P}_{\mathrm{n}}\right)\right)=2$ and $\mathcal{J}^{*}\left(\mathrm{~L}\left(\mathrm{P}_{\mathrm{n}}\right)\right)=3$.

(ii) $\mathcal{J}\left(\mathrm{M}\left(\mathrm{P}_{2}\right)\right)=2$ and $\mathrm{M}\left(\mathrm{P}_{\mathrm{n}}\right) \mathrm{n} \geq 3$ does not admit a J-coloring and $\mathcal{J}^{*}\left(M\left(P_{n}\right)\right)=3$.

(iii) $\mathcal{J}\left(\mathrm{T}\left(\mathrm{P}_{\mathrm{n}}\right)\right)=\mathcal{J}^{*}\left(\mathrm{~T}\left(\mathrm{P}_{\mathrm{n}}\right)\right)=3$.

(iv) For connectivity, let $\mathrm{n} \geq 5$. Then $\mathcal{J}\left(\mathrm{J}\left(\mathrm{P}_{5}\right)\right)=3$ and $\mathcal{J}^{*}\left(\mathrm{~J}\left(\mathrm{P}_{5}\right)\right)=3$ and for $\mathrm{n} \geq 6$,

$$
\mathcal{J}\left(J\left(P_{n}\right)\right)=\mathcal{J}^{*}\left(J\left(P_{n}\right)\right)= \begin{cases}\frac{\mathrm{n}}{2} & \mathrm{n} \text { is even } \\ \left\lfloor\frac{\mathrm{n}}{2}\right\rfloor & \mathrm{n} \text { is odd }\end{cases}
$$

(v) $\mathcal{J}\left(\mathrm{C}\left(\mathrm{P}_{\mathrm{n}}\right)\right)=\mathcal{J}^{*}\left(\mathrm{C}\left(\mathrm{P}_{\mathrm{n}}\right)\right)=3$.

\section{Proof.}

(i) Since $L\left(P_{n}\right)=P_{n-1}$, the result follows from the result that for any connected graph $G, \mathcal{J}(\mathrm{G}) \leq \delta(\mathrm{G})+1$.

(ii) Since $M\left(P_{2}\right)=P_{3}$ the result follows from the result that for any connected graph $G, \mathcal{J}(G) \leq \delta(G)+1$. For $n \geq 3$, the middle graph contains a triangle hence, $\mathcal{J}\left(M\left(P_{n}\right)\right) \geq \chi\left(M\left(P_{n}\right)\right) \geq 3$. Also $M\left(P_{n}\right)$ has two pendant vertices therefore $r_{\chi}\left(M\left(P_{n}\right)\right) \neq n$. So $M\left(P_{n}\right), n \geq 3$ does not admit a Jcoloring. The derivative graph $\mathrm{G}^{\prime}=\mathrm{M}\left(\mathrm{P}_{\mathrm{n}}\right)-\left\{v_{1}, v_{n}\right\}$ contains a triangle and $\delta\left(G^{\prime}\right)=2$. Therefore, $\mathcal{J}^{*}\left(M\left(P_{n}\right)\right)=3$.

(iii) Since $\mathcal{J}\left(T\left(P_{n}\right)\right) \leq \delta\left(\mathcal{J}\left(T\left(P_{n}\right)\right)+1=3\right.$ and $T\left(P_{n}\right)$ contains a triangle, $\mathcal{J}\left(T\left(P_{n}\right)\right)=3$. As $T\left(P_{n}\right)$ has no pendant vertex and contains an odd cycle $C_{3}$, the result $\mathcal{J}^{*}\left(T\left(P_{n}\right)\right)=3$ is immediate.

(iv) For $P_{5}$ we have $J\left(P_{5}\right)=P_{4}$. Hence, the result follows from for any connected graph $\mathrm{G}, \mathcal{J}(\mathrm{G}) \leq \delta(\mathrm{G})+1$. For a path $\mathrm{P}_{\mathrm{n}}, \mathrm{n} \geq 6$ and edge set consecutively labeled as $e_{1}, e_{2}, e_{3}, \ldots, e_{n-1}$ and the corresponding line graph vertices consecutively labeled as $\mathfrak{u}_{1}, \mathfrak{u}_{2}, \mathfrak{u}_{3}, \ldots, \mathfrak{u}_{n-1}$, we 
have the consecutive vertex $\chi^{-}$-coloring sequence of $J\left(P_{n}\right)$ is given by $c_{1}, c_{1}, c_{2}, c_{2}, c_{3}, c_{3}, \ldots, c_{\frac{n}{2}}$ if $n$ is even and $c_{1}, c_{1}, c_{2}, c_{2}, c_{3}, c_{3}, \ldots, c_{\left\lfloor\frac{n}{2}\right\rfloor}, c_{\left\lfloor\frac{n}{2}\right\rfloor}$ if $n$ is odd. Since the vertices $u_{i}, u_{i+1}, 1 \leq i \leq n-2$ are pairwise not adjacent, the $\chi^{-}$-coloring is maximal as well. Clearly, every vertex $u_{i}$ yields a rainbow neighbourhood. Therefore, the result follows.

(v) Since $C\left(P_{n}\right)$ has no pendant vertex and contains an odd cycle $C_{5}$, the result is immediate.

Next, we consider cycles $C_{n}, n \geq 3$. In [8], it is proved that

Theorem 14 [8] If $\mathrm{C}_{\mathrm{n}}$ admits a J-coloring then:

$$
\mathcal{J}\left(\mathrm{C}_{n}\right)= \begin{cases}3 & \text { if } \mathrm{n} \equiv 0(\bmod 3) \\ 2 & \text { if } \mathrm{n} \equiv 0(\bmod 2) \text { and } \mathrm{n} \not \equiv 0(\bmod 3) .\end{cases}
$$

Analogous to the proof of Theorem 2.7 in [8], we now establish the corresponding results for the derivatives of cycle graphs in the following proposition.

Proposition 15 For a cycle $\mathrm{C}_{\mathrm{n}}, \mathrm{n} \geq 3$ and edge set consecutively labeled as $\mathrm{e}_{1}, \mathrm{e}_{2}, \mathrm{e}_{3}, \ldots, \mathrm{e}_{\mathrm{n}}$ and the corresponding line graph vertices consecutively labeled as $\mathrm{u}_{1}, \mathrm{u}_{2}, \mathfrak{u}_{3}, \ldots, \mathfrak{u}_{\mathrm{n}}$, we have

(i) $\mathcal{J}\left(\mathrm{L}\left(\mathrm{C}_{\mathrm{n}}\right)\right)=\mathrm{J}^{*}\left(\mathrm{~L}\left(\mathrm{C}_{\mathrm{n}}\right)\right)=2$ if and only if $\mathrm{n} \equiv \mathrm{O}(\bmod 2)$ and $\mathrm{n} \not \equiv$ $\mathrm{O}(\bmod 3)$, and $\mathcal{J}\left(\mathrm{L}\left(\mathrm{C}_{n}\right)\right)=\mathcal{J}^{*}\left(\mathrm{~L}\left(\mathrm{C}_{\mathrm{n}}\right)\right)=3$ if and only if $\mathrm{n} \equiv \mathrm{O}(\bmod 3)$, else, $\mathrm{L}\left(\mathrm{C}_{\mathrm{n}}\right)$ does not admit a J-coloring.

(ii) For $\mathrm{n} \geq 3, \mathcal{J}\left(M\left(C_{n}\right)\right)=\mathcal{J}^{*}\left(M\left(C_{n}\right)\right)=3$ if $n \equiv 0(\bmod 3)$, or if, $M\left(C_{n}\right)$ for $\mathrm{n} \neq \equiv 0(\bmod 3)$, and without loss of generality admits the coloring: $\mathrm{c}\left(v_{1}\right)=\mathrm{c}_{1}, \mathrm{c}\left(\mathrm{u}_{1}\right)=\mathrm{c}_{2}, \mathrm{c}\left(v_{2}\right)=\mathrm{c}_{3}, \mathrm{c}\left(\mathrm{u}_{2}\right)=\mathrm{c}_{1}, \mathrm{c}\left(v_{3}\right)=\mathrm{c}_{2}, \mathrm{c}\left(\mathrm{u}_{3}\right)=$ $\mathrm{c}_{3}, \ldots, \mathrm{c}\left(v_{n-1}\right)=\mathrm{c}_{1}, \mathrm{c}\left(\mathrm{u}_{n-1}\right)=\mathrm{c}_{2}, \mathrm{c}\left(v_{n}\right)=\mathrm{c}_{1}, \mathrm{c}\left(\mathrm{u}_{\mathrm{n}}\right)=\mathrm{c}_{3}$, else, $\mathrm{M}\left(\mathrm{C}_{\mathrm{n}}\right)$ does not admit a J-coloring.

(iii) $\mathcal{J}\left(\mathrm{T}\left(\mathrm{C}_{\mathrm{n}}\right)\right)=\mathrm{J}^{*}\left(\mathrm{~T}\left(\mathrm{C}_{\mathrm{n}}\right)\right)=4$ if and only if $\mathrm{n}$ is even, else, $\mathrm{T}\left(\mathrm{C}_{\mathrm{n}}\right)$ does not admit a J-coloring.

(iv) For $\mathrm{n} \geq 6, \mathcal{J}\left(\mathrm{J}\left(\mathrm{C}_{\mathrm{n}}\right)\right)=\mathcal{J}^{*}\left(\mathrm{~J}\left(\mathrm{C}_{\mathrm{n}}\right)\right)=\left\{\begin{array}{ll}\frac{\mathrm{n}}{2} & \mathrm{n} \text { is even } \\ \left\lfloor\frac{\mathrm{n}}{2}\right\rfloor & \mathrm{n} \text { is odd. }\end{array}\right.$.

(v) $\mathcal{J}\left(C\left(C_{n}\right)\right)=\mathcal{J} *\left(C\left(C_{n}\right)\right)=3$. 
Proof. (i) Because $\mathrm{L}\left(\mathrm{C}_{n}\right)=\mathrm{C}_{n}$ the result follows from Corollary 3.6. Also because $L\left(C_{n}\right)$ has no pendant edges, $\mathcal{J}\left(L\left(C_{n}\right)\right)=\mathcal{J}^{*}\left(L\left(C_{n}\right)\right)$.

(ii) If $M\left(C_{n}\right)$ admits a J-coloring then $\mathcal{J}\left(M\left(C_{n}\right)\right) \leq \delta\left(\mathcal{J}\left(M\left(C_{n}\right)\right)+1=3\right.$. For $\mathrm{n} \equiv 0(\bmod 3)$, consider the coloring: $\mathrm{c}\left(v_{1}\right)=\mathrm{c}_{1}, \mathrm{c}\left(\mathrm{u}_{1}\right)=\mathrm{c}_{2}, \mathrm{c}\left(v_{2}\right)=\mathrm{c}_{3}$, $c\left(u_{2}\right)=c_{1}, c\left(v_{3}\right)=c_{2}, c\left(u_{3}\right)=c_{3}, \ldots, c\left(u_{n-1}\right)=c_{1}, c\left(v_{n}\right)=c_{2}, c\left(u_{n}\right)=c_{3}$.

From the definition of the middle graph, we know that $M\left(C_{n}\right)$ has $n$ triangles stringed so clearly the proper coloring is maximum and all vertices yield a rainbow neighbourhood. Part 2 follows by similar reasoning and hence the result follows. Also, since $M\left(C_{n}\right)$ has no pendant edges, $\mathcal{J}\left(M\left(C_{n}\right)\right)=\mathcal{J}^{*}\left(M\left(C_{n}\right)\right)$. In all other cases, $\chi\left(\left(M\left(C_{n}\right)\right)=4\right.$ and a J-coloring does not exist.

(iii) Note that $\mathcal{J}\left(T\left(C_{n}\right)\right) \leq \delta\left(\mathcal{J}\left(T\left(C_{n}\right)\right)+1=5\right.$. Since $T\left(C_{n}\right)$ contains a triangle, $\mathcal{J}\left(T\left(C_{n}\right)\right) \geq 3$. Furthermore, $\chi\left(\left(T\left(C_{n}\right)\right)=4\right.$ if and only if $n \equiv 0(\bmod 2)$ and $\mathrm{n} \not \equiv 0(\bmod 3)$, and all vertices yield a rainbow neighbourhood. Also, for any set of vertices $\mathrm{V}^{\prime}=\left\{v_{i}, v_{i+1}, v_{i+2}, v_{i+2}, v_{i+3}, v_{i+4}\right\} \mapsto\left\{v_{i} v_{j}: 1 \leq i \leq\right.$ $\mathrm{n}, 0 \leq j \leq 4$, and $(i+j) \mapsto(i+j)(\bmod 6)\}$, the induced subgraph $\left\langle\mathrm{V}^{\prime}\right\rangle \neq \mathrm{K}_{5}$. Therefore, $\mathcal{J}\left(\mathrm{T}\left(\mathrm{C}_{n}\right)\right)=4$. Also because $\mathrm{T}\left(\mathrm{C}_{n}\right)$ has no pendant edges, $\mathcal{J}\left(T\left(C_{n}\right)\right)=\mathcal{J}^{*}\left(T\left(C_{n}\right)\right)$. Otherwise, $\chi\left(\left(T\left(C_{n}\right)\right)=5\right.$, and not all vertices yield a rainbow neighbourhood and hence a J-coloring is not obtained.

(iv) For $\mathrm{n}=5, \mathrm{~J}\left(\mathrm{C}_{5}\right)=\mathrm{C}_{5}$ and thus, does not admit a J-coloring. For a path $C_{n}, n \geq 6$ and edge set consecutively labeled as $e_{1}, e_{2}, e_{3}, \ldots, e_{n-1}$ and the corresponding line graph vertices consecutively labeled as $\mathfrak{u}_{1}, \mathfrak{u}_{2}, \mathfrak{u}_{3}, \ldots, \mathfrak{u}_{n-1}$, we have the consecutive vertex $\chi^{-}$-coloring sequence of $J\left(C_{n}\right)$ is given by

$c_{1}, c_{1}, c_{2}, c_{2}, c_{3}, c_{3}, \ldots, c_{\frac{n}{2}}$ if $n$ is even and $c_{1}, c_{1}, c_{2}, c_{2}, c_{3}, c_{3}, \ldots, c_{\left\lfloor\frac{n}{2}\right\rfloor}, c_{\left\lfloor\frac{n}{2}\right\rfloor}$ if $n$ is odd ( $n-1$ entries). As the vertices $u_{i}, u_{i+1}, 1 \leq i \leq n-2$ are pairwise not adjacent, the $\chi^{-}$-coloring is maximal as well. Clearly, every vertex $u_{i}$ yields a rainbow neighbourhood. Therefore, the result follows.

(v) The result is trivial for $\mathrm{C}\left(\mathrm{C}_{3}\right)$. For $n \geq 4, \mathcal{J}\left(C\left(C_{n}\right)\right) \leq \delta\left(\mathcal{J}\left(C\left(C_{n}\right)\right)+1=3\right.$. Since $\chi\left(\left(C\left(C_{n}\right)\right)=3\right.$ and all vertices yield a rainbow neighbourhood and $C\left(C_{n}\right)$ contains a cycle $\mathrm{C}_{5}$, the result $\mathcal{J}\left(\mathrm{C}\left(\mathrm{C}_{n}\right)\right)=3$ holds immediately. Also, since $C\left(C_{n}\right)$ has no pendant edges, $\mathcal{J}\left(C\left(C_{n}\right)\right)=\mathcal{J}^{*}\left(C\left(C_{n}\right)\right)$.

\section{Extremal results for certain graphs}

For a graph $\mathrm{G}$ of order $\mathrm{n} \geq 1$, which admits a J-coloring the minimum (or maximum) number of edges in a subset $E_{k}^{\prime} \subseteq E(G)$ whose removal ensures that $\mathcal{J}\left(G-E_{k}^{\prime}\right)=k, 1 \leq k \leq \mathcal{J}(G)$, is discussed in this section. These extremal variables are called the minimum (or maximum) rainbow bonding variables and are denoted $r_{k}^{-}(G)$ and $r_{k}^{+}(G)$, respectively. A graph $G$ which does not 
admit a J-coloring has $r_{k}^{-}(G)$ and $r_{k}^{+}(G)$ undefined. For such aforesaid graph it is always possible to remove a minimal set of edges, $E^{\prime \prime}$, which is not necessarily unique such that $G-E^{\prime \prime}$ admits a J-coloring. This is formalised in the next result.

Lemma 16 For any connected graph $\mathrm{G}$ which does not admit a J-coloring, a minimal set of edges, $\mathrm{E}^{\prime \prime}$ which is not necessarily unique, can be removed such that $\mathrm{G}-\mathrm{E}^{\prime \prime}$ admits a J-coloring.

Proof. Since any connected graph $G$ of order $n$ and size $\varepsilon(G)=p$ has a spanning subtree and any tree admits a J-coloring, at most $p-(n-1)$ edges must be removed from $G$. Therefore, if $p-(n-1)$ is not a minimal number of edges to be removed then a minimal set of edges $E^{\prime},\left|E^{\prime}\right|<p-(n-1)$ must exist whose removal results in a spanning subgraph $\mathrm{G}^{\prime}$ which allows a J-coloring.

It is obvious from Lemma 16 that the restriction of connectedness can be relaxed if $G=\bigcup H_{i}, 1 \leq i \leq t$ and it is possible that $\mathcal{J}\left(H_{i}-E^{\prime \prime}\right)_{\forall i}=k$, $k$ some integer constant.

It is obvious that for a complete graph $K_{n}, \mathcal{J}\left(K_{n}\right)=n$. To ensure $\mathcal{J}\left(K_{n}\right)=n$, no edges can be removed. Therefore, $r_{n}^{-}\left(K_{n}\right)=r_{n}^{+}\left(K_{n}\right)=0$.

Theorem 17 For a complete graph $\mathrm{K}_{\mathrm{n}}, \mathrm{n} \geq 1$ we have

(i) For $\mathrm{n}$ is even and $\frac{\mathrm{n}}{2} \leq \mathrm{k} \leq \mathrm{n}$ and $\mathcal{J}\left(\mathrm{K}_{\mathrm{n}}-\mathrm{E}_{\mathrm{k}}^{\prime}\right)=\mathrm{k}$, then $\mathrm{r}_{\mathrm{k}}^{-}\left(\mathrm{K}_{\mathrm{n}}\right)=\mathrm{n}-\mathrm{k}$.

(ii) For $\mathrm{n}$ is odd and $\left\lceil\frac{\mathrm{n}}{2}\right\rceil \leq \mathrm{k} \leq \mathrm{n}$, and $\mathcal{J}\left(\mathrm{K}_{\mathrm{n}}-\mathrm{E}_{\mathrm{k}}^{\prime}\right)=\mathrm{k}$, then $\mathrm{r}_{\mathrm{k}}^{-}\left(\mathrm{K}_{\mathrm{n}}\right)=\mathrm{n}-\mathrm{k}$.

(iii) For $\mathrm{n} \in \mathbb{N}$ and $1 \leq \mathrm{k} \leq \mathrm{n}$, and $\mathcal{J}\left(\mathrm{K}_{\mathrm{n}}-\mathrm{E}_{\mathrm{k}}^{\prime}\right)=\mathrm{k}$, then $\mathrm{r}_{\mathrm{k}}^{+}\left(\mathrm{K}_{\mathrm{n}}\right)=\frac{1}{2}(\mathrm{n}+$ $1-k)(n-k)$.

Proof. (i) For $n$ is even and $\frac{n}{2} \leq k \leq n$, exactly 0 or 1 or 2 or 3 or $\cdots$ or $\frac{n}{2}$ edges between distinct pairs of vertices can be removed to obtain $\mathcal{J}\left(\mathrm{K}_{n}-\right.$ $\left.E_{k}^{\prime}\right)=n, n-1, n-2, \ldots, \frac{n}{2}$. Hence, $r_{k}^{-}\left(K_{n}\right)=0,1,2,3, \ldots, \frac{n}{2}$. In other words $r_{k}^{-}\left(K_{n}\right)=n-k, \frac{n}{2} \leq k \leq n$.

(ii) The result follows through similar reasoning as that in (i).

(iii) In any clique of order $t$, the removal of the $\frac{1}{2} t(t-1)$ edges is the maximum number of edges whose removal renders $\mathcal{J}\left(\mathfrak{N}_{\mathfrak{t}}\right)=1$ hence, all vertices can be colored say, $\mathbf{c}_{1}$. Through immediate mathematical induction it follows that we iteratively remove the maximum number of edges $r_{k}^{+}\left(K_{n}\right)=$ $0,1,3,6,10, \ldots, \frac{1}{2}(n+1-k)(n-k), 1 \leq k \leq n$ of cliques $K_{1}, K_{2}, K_{3}, \ldots, K_{n}$ to obtain $\mathcal{J}\left(K_{n}-E_{k}^{\prime}\right)=n, n-1, n-2, \ldots, 1$. Hence, the result follows. 
Theorem 18 A graph $\mathrm{G}$ of order $\mathrm{n}$ which allows a J-coloring, has $\mathrm{r}_{\mathrm{k}}^{-}(\mathrm{G})=$ $\mathrm{r}_{\mathrm{k}}^{+}(\mathrm{G})$ if and only if $\mathrm{J}(\mathrm{G})=2$.

Proof. If $\mathcal{J}(G)=2$ then all edges are incident with colors $c_{1}, c_{2}$. Therefore all edges must be removed to obtain the null graph $\mathfrak{N}_{0}$ for which $\mathcal{J}\left(\mathfrak{N}_{0}\right)=1$. Hence, $r_{k}^{-}(G)=r_{k}^{+}(G)$.

Conversely, let $r_{k}^{-}(G)=r_{k}^{+}(G)$. Then, assume that at least one edge say, $e$ is incident with color $c_{3}$. It implies that $\mathrm{G}$ contains at least a triangle or an odd cycle. Therefore, $\varepsilon(G) \geq 3$. To ensure a proper coloring on removing edge $e$ the color $c_{3}$ must change to either $c_{1}$ or $c_{2}$ which is always possible. If $\mathcal{J}(G-e)=2$ then $r_{k}^{+}(G)=1$ which is a contradiction because any one additional edge may have been removed, implying $r_{k}^{+}(G) \geq 2$. For colors $c_{4}, c_{5}, c_{6}, \ldots, \mathcal{J}(G)$, similar contradictions follows through immediate induction. Therefore, if $r_{k}^{-}(G)=$ $\mathrm{r}_{\mathrm{k}}^{+}(\mathrm{G})$ then, $\mathcal{J}(\mathrm{G})=2$.

\section{Conclusion}

Clearly the cycles for which the the middle graphs admit a J-coloring in accordance with the second part of Proposition 13(ii) require to be characterised if possible. It follows from Theorem 18 that for the cases $n$ is even and $1 \leq k<\frac{n}{2}$, or $n$ is odd and $1 \leq k<\left\lceil\frac{n}{2}\right\rceil$, determining $r_{k}^{-}\left(K_{n}\right)$ remains open. It is suggested that an algorithm must be described to obtain these values.

Example 19 For the complete graph $K_{9}$ with vertices $v_{1}, v_{2}, v_{3}, \ldots, v_{9}$, Theorem $17\left(\right.$ ii) admits the minimum removal of $r_{n, k}^{-}\left(K_{n}\right)=4$ edges to obtain $\mathcal{J}\left(K_{n}-E_{k}^{\prime}\right)=5$. Without loss of generality say the edges were. $v_{1} v_{2}, v_{3} v_{4}, v_{6} v_{6}$, $v_{7} v_{8}$. To obtain $\mathcal{J}\left(\mathrm{K}_{\mathrm{n}}-\mathrm{E}_{\mathrm{k}}^{\prime}\right)=4$ we only remove without loss of generality say, the edges $v_{7} v_{9}, v_{8} v_{9}$. To obtain $\mathcal{J}\left(K_{n}-E_{k}^{\prime}\right)=3$ we only remove without loss of generality say, the edges $v_{1} v_{3}, v_{1} v_{4}, v_{2} v_{3}, v_{2} v_{4}$. To obtain $\partial\left(K_{n}-E_{k}^{\prime}\right)=2$ we only remove without loss of generality say, the edges $v_{5} v_{7}, v_{5} v_{8}, v_{5} v_{9}, v_{6} v_{7}$, $v_{6} v_{8}, v_{6} v_{9}$. To obtain $\mathcal{J}\left(\mathrm{K}_{\mathrm{n}}-\mathrm{E}_{\mathrm{k}}^{\prime}\right)=1$ we remove all remaining edges. It implies that as $\mathcal{J}\left(K_{n}-E_{k}^{\prime}\right)$ iteratively ranges through the values $5,4,3,2,1$ the value of $r_{k}^{-}\left(K_{9}\right)$ ranges through, 4, 6, 10, 16, 36 .

Determining the range of minimum (maximum) rainbow bonding variables for other classes of graphs is certainly worthy research. For a graph $G$ which does not allow a J-coloring it follows from Lemma 16 that a study of $r_{k}^{-}\left(G^{\prime}\right)$ and $r_{k}^{+}\left(G^{\prime}\right)$ with $G^{\prime}$ a maximal spanning subgraph of $G$ which does allow a J-coloring, is open. 


\section{References}

[1] J. A. Bondy and U. S. R. Murty, Graph Theory, Springer, New York, 1976. $\Rightarrow 95$

[2] G. Chartrand and P. Zhang, Chromatic Graph Theory, CRC Press, 2009. $\Rightarrow 95$

[3] F. Harary, Graph Theory, New Age International, New Delhi, 2001. $\Rightarrow$ 95, 96

[4] T. R. Jensen and B. Toft, Graph Coloring Problems, John Wiley \& Sons, 1995. $\Rightarrow 95$

[5] J. Kok, N. K. Sudev and M. K. Jamil, Rainbow neighbourhood number of graphs, Proy. J. Math., 38, 3 (2019) 471-487. $\Rightarrow 96,99$

[6] S. Naduvath, S. Chandoor, S.J. Kalayathankal, J Kok, A note on the rainbow neighbourhood number of graphs, Nat. Acad. Sci. Letters, 42, 2 (2019) $135-138 . \Rightarrow 96$

[7] S. Naduvath, S. Chandoor, S. J. Kalayathankal, J Kok, Some new results on the rainbow neighbourhood number of graphs, Nat. Acad. Sci. Letters, 42, 3 (2019) 249-252. $\Rightarrow 96$

[8] N. K. Sudev, On certain J-coloring parameters of graphs, Nat. Acad. Sci. Letters, (2019), in press. $\Rightarrow 96,97,100,103,104$

[9] D. B. West, Introduction to Graph Theory, Pearson Education Inc., Delhi, 2001. $\Rightarrow 95$

Received: July 4, 2019•Revised: August 6, 2019 